\title{
A uniformly spread measure criterion
}

\author{
A. Dudko, S. Favorov
}

\begin{abstract}
We prove that if all shifts of a measure in the Euclidean space are close in a sense to each other, then this measure is close to the Lebesgue one.
\end{abstract}

Let $\left(a_{n}\right)_{n \in \mathbb{N}}$ be a discrete sequence in $\mathbb{R}^{d}$, i.e., a map $\mathbb{N} \rightarrow \mathbb{R}^{d}$ such that its image $\left\{a_{n}\right\}_{n \in \mathbb{N}}$ has no limit point in $\mathbb{R}^{d}$ and each $x \in\left\{a_{n}\right\}_{n \in \mathbb{N}}$ has at most a finite multiplicity. Following Laczkovich [1], [2], we say that the sequence is uniformly spread over $\mathbb{R}^{d}$, if there is $\alpha>0$ such that

$$
\inf _{\psi} \sup _{n}\left|a_{n}-\alpha \psi(n)\right|<\infty
$$

where the infimum is taken over all bijections $\psi: \mathbb{N} \rightarrow \mathbb{Z}^{d}$.

Using the idea of the mass transfer (see, for example, [4]), M. Sodin, B. Tsirelson extended the above definition to measures in $\mathbb{R}^{d}$. In [3] they introduce a transportation distance between arbitrary locally finite positive measures $\nu_{1}$ and $\nu_{2}$

$$
\operatorname{Tra}\left(\nu_{1}, \nu_{2}\right)=\inf _{\gamma} \sup \{|x-y|: x, y \in \overline{\operatorname{supp} \gamma}\}
$$

Here the infimum is taken over all transportation measures $\gamma$ between measures $\nu_{1}$ and $\nu_{2}$, where the latter means

$$
\begin{gathered}
\iint_{\mathbb{R}^{d} \times \mathbb{R}^{d}} \varphi(x) d \gamma(x, y)=\int_{\mathbb{R}^{d}} \varphi(x) d \nu_{1}(x), \\
\iint_{\mathbb{R}^{d} \times \mathbb{R}^{d}} \varphi(y) d \gamma(x, y)=\int_{\mathbb{R}^{d}} \varphi(y) d \nu_{2}(y)
\end{gathered}
$$

for all continuous functions $\varphi: \mathbb{R}^{d} \rightarrow \mathbb{R}$ with a compact support. 
The degree of concentration of $\gamma$ near diagonal of $\mathbb{R}^{d} \times \mathbb{R}^{d}$ shows the closeness of $\nu_{1}$ and $\nu_{2}$ to each other.

A continuous analogue of definition of uniformly spreading (1) (actually belonging to M. Sodin, B. Tsirelson [3] ) has the form

Definition 1. A locally finite positive measure $\nu$ on $\mathbb{R}^{d}$ is uniformly spread over $\mathbb{R}^{d}$, if there is $\beta>0$ such that

$$
\operatorname{Tra}(\nu, \beta \omega)<\infty
$$

where $\omega$ is the Lebesgue measure on $\mathbb{R}^{d}$.

In what follows we denote by $\nu^{x}$ the shift of the measure $\nu$ along $x \in \mathbb{R}^{d}$, and for any $x=\left(x^{1}, \ldots, x^{d}\right) \in \mathbb{R}^{d}$ put

$$
Q(x, r)=\left\{y=\left(y^{1}, \ldots, y^{d}\right) \in \mathbb{R}^{d}: x^{j}-r / 2 \leq y^{j}<x^{j}+r / 2, j=1, \ldots, d\right\} .
$$

Also, we denote by $\chi^{m}(y), m \in \mathbb{Z}^{d}$, the indicator of the cube $Q(m, 1)$.

For a discrete sequence $\left(a_{n}\right)_{n \in \mathbb{N}}$ we set

$$
\nu=\sum_{n} \delta^{a_{n}}
$$

where $\delta$ is a unit mass sitting in the origin. Then

$$
C_{1} \operatorname{Tra}\left(\nu, \alpha^{-d} \omega\right) \leq \inf _{\psi} \sup _{n}\left|a_{n}-\alpha \psi(n)\right| \leq C_{2} \operatorname{Tra}\left(\nu, \alpha^{-d} \omega\right)
$$

As above, the infimum is taken over all bijections $\psi: \mathbb{N} \rightarrow \mathbb{Z}^{d}$, and the constants $C_{1}$ and $C_{2}$ depend only on the dimension $d$.

In fact, if the sequence satisfies (1), then the measure $\gamma=\sum \delta^{a_{n}}(x) \otimes$ $\alpha^{-d} \chi^{\alpha \psi(n)}(y / \alpha) \omega(y)$ is a transportation measure between $\nu$ and $\alpha^{-d} \omega$, and the first inequality in (6) follows easily. The second inequality in (6) is nontrivial. Its proof in [2] is based on the Rado Lemma from the graph theory.

The main result of our article is the following theorem.

Theorem 1. A positive locally finite measure $\nu \not \equiv 0$ is uniformly spread over $\mathbb{R}^{d}$ if and only if there exists a constant $C_{3}<\infty$ such that

$$
\operatorname{Tra}\left(\nu, \nu^{z}\right)<C_{3} \quad \forall z \in \mathbb{R}^{d}
$$


Proof of the Theorem 1, By [3, Theorem 1.2], we have

$$
\operatorname{Tra}\left(\nu_{1}, \nu_{3}\right) \leq \operatorname{Tra}\left(\nu_{1}, \nu_{2}\right)+\operatorname{Tra}\left(\nu_{2}, \nu_{3}\right) .
$$

Hence, (4) and the equality $\omega^{z} \equiv \omega$ imply (7).

Next, suppose that the measure $\nu$ satisfies (7). We decompose $\mathbb{R}^{d}$ into the cubes $Q(m, 1), m \in \mathbb{Z}^{d}$. Let $\gamma_{m}$ be a transportation measure between $\nu$ and $\nu^{m}$. For fixed $k \in \mathbb{Z}^{d}$ set

$$
\lambda_{k, n}=\frac{1}{(2 n+1)^{d}} \sum_{\|m-k\|_{\infty} \leq n} \gamma_{m} .
$$

It follows from definition of a transportation measure (2) and (3) that $\lambda_{k, n}$ is a transportation measure between $\nu$ and

$$
\mu_{k, n}=\frac{1}{(2 n+1)^{d}} \sum_{\|m-k\|_{\infty} \leq n} \nu^{m} .
$$

Note that we can replace in (2) and (3) the function $\varphi$ by the indicator function of any bounded Borel subset of $\mathbb{R}^{d}$. Therefore, by (7), we get for any $m, k \in \mathbb{Z}^{d}$

$$
\begin{aligned}
\nu^{m}(Q(k, 1)) & =\nu^{m+k}(Q(0,1))=\gamma_{m+k}\left(Q(0,1) \times \mathbb{R}^{d}\right) \\
& \leq \gamma_{m+k}\left(\mathbb{R}^{d} \times Q\left(0, C_{3}+1\right)\right)=\nu\left(Q\left(0, C_{3}+1\right)\right) .
\end{aligned}
$$

Hence the measures $\mu_{k, n}$ are uniformly bounded on every compact subset of $\mathbb{R}^{d}$, and the measures $\lambda_{k, n}$ are uniformly bounded on every compact subset of $\mathbb{R}^{d} \times \mathbb{R}^{d}$. By (7),

$$
\operatorname{Tra}\left(\nu, \mu_{k, n}\right) \leq \sup _{m \in \mathbb{Z}^{d}} \operatorname{Tra}\left(\nu, \nu^{m}\right) \leq C_{3} .
$$

Take a subsequence $n^{\prime} \rightarrow \infty$ such that for each $k \in \mathbb{Z}^{d}$ the measures $\lambda_{k, n}$ weakly converge to some measure $\lambda_{k}$, and the measures $\mu_{k, n}$ weakly converge to some measure $\mu_{k}$. Note that for any $k, k^{\prime} \in \mathbb{Z}^{d}$

$\mu_{k, n}-\mu_{k^{\prime}, n}=\frac{1}{(2 n+1)^{d}}\left[\sum_{\|m-k\|_{\infty} \leq n,\left\|m-k^{\prime}\right\|_{\infty}>n} \nu^{m}-\sum_{\left\|m-k^{\prime}\right\|_{\infty} \leq n,\|m-k\|_{\infty}>n} \nu^{m}\right]$,

and

$$
\operatorname{card}\left\{m:\|m-k\|_{\infty} \leq n,\left\|m-k^{\prime}\right\|_{\infty}>n\right\}=O\left(n^{d-1}\right),
$$




$$
\operatorname{card}\left\{\left\|m-k^{\prime}\right\|_{\infty} \leq n,\|m-k\|_{\infty}>n\right\}=O\left(n^{d-1}\right),
$$

as $n \rightarrow \infty$. Hence, for each $k, k^{\prime} \in \mathbb{Z}^{d}$ the variations of measures $\mu_{k, n}-\mu_{k^{\prime}, n}$ on every compact subset tend to zero as $n \rightarrow \infty$. The same assertion is valid for the differences $\lambda_{k, n}-\lambda_{k^{\prime}, n}$. Therefore, for all $k \in \mathbb{Z}^{d}$ we have $\mu_{k} \equiv \mu$ and $\lambda_{k} \equiv \lambda$ for some measures $\lambda$ and $\mu$. Moreover,

$$
\mu(Q(k, 1))=\mu\left(Q\left(k^{\prime}, 1\right)\right) \quad \forall k, k^{\prime} \in \mathbb{Z}^{d} .
$$

It can easily be checked that the measure $\lambda$ is a transportation measure between $\nu$ and $\mu$. By (8), we get

$$
\operatorname{Tra}(\nu, \mu) \leq C_{3}
$$

Hence, $\mu \not \equiv 0$. Let $\rho_{m}$ be the restriction of the measure $\mu$ to $Q(m, 1)$. We obtain that the measure

$$
\sum_{m \in \mathbb{Z}^{d}} \rho_{m}(x) \otimes \chi^{m}(y) \omega(y)
$$

is a transportation measure between $\mu$ and $\beta \omega$ with $\beta=\mu(Q(0,1))$. Finally,

$$
\operatorname{Tra}(\nu, \beta \omega) \leq \operatorname{Tra}(\nu, \mu)+\operatorname{Tra}(\mu, \beta \omega) \leq C_{3}+1
$$

Theorem 1 is proved.

For discrete sequences in $\mathbb{R}^{d}$ we obtain the following result.

Theorem 2. A discrete sequence $\left(a_{n}\right)_{n \in \mathbb{N}} \subset \mathbb{Z}^{d}$ satisfies (11) if and only if for any $z \in \mathbb{R}^{d}$ there is a bijection $\sigma: \mathbb{N} \rightarrow \mathbb{N}$ such that

$$
\sup _{n}\left|a_{n}+z-a_{\sigma(n)}\right| \leq C_{7}<\infty .
$$

Proof. Clearly, (11) yields (9). On the other hand, if (9) holds and $\nu$ is the measure defined in (5), then the measure

$$
\sum_{n \in \mathbb{N}} \delta^{a_{n}+z}(x) \otimes \delta^{a_{\sigma(n)}}(y)
$$

is a transportation measure between $\nu^{z}$ and $\nu$. Condition (9) implies $\operatorname{Tra}\left(\nu^{z}, \nu\right)<\infty$. By Theorem 1, for some $\alpha>0$ we have $\operatorname{Tra}\left(\nu, \alpha^{-d} \omega\right)<\infty$. Using (6), we obtain (11). 


\section{References}

[1] M. LACZKovich Equidecoposability and discrepancy: a solution of Tarski's circle-squaring problem. J. Reine Angew.Math. V.404 (1990), 77-117.

[2] M. Laczkovich Uniformly spread discrete sets in $\mathbb{R}^{d}$, J.London Math. Soc.(2), 46. (1992), 39-57.

[3] M. Sodin, B. Tsirelson Uniformly spread measures and vector fields, arXiv: 0801.2505

[4] L. V. Kantorovoch, G. P. Akilov Functional analysis in normed spaces Pergamon Press, Oxford-Elmsford, N.Y., 1982.

Mathematical School, Kharkov national university, Swobody sq.4, Kharkov, 61077 Ukraine

e-mail: Sergey.Ju.Favorov@univer.kharkov.ua

Mathematics Division, Institute for Low Temperature Physics and Engineering, 47 Lenin ave., Kharkov 61103, Ukraine

e-mail: artemdudko@rambler.ru 